\title{
Structural Stochastic Multiresonance in Hierarchical Networks
}

\author{
A. KRAWIECKI AND M. Kaim
}

Faculty of Physics, Warsaw University of Technology, Koszykowa 75, PL-00-662 Warsaw, Poland

Systems with a structure of hierarchical networks, consisting of simple units placed in the nodes and interacting along the edges of the network, are ubiquitous in the modern society and economy. In this paper the problem of signal detection and transmission in such systems in the presence of noise is analyzed from the point of view of stochastic resonance. As examples simple tree-like and more complex Ravasz-Barabási networks of interacting threshold elements are considered. It is shown that stochastic multiresonance is often observed, a phenomenon characterized by the presence of two or more maxima of the output signal-to-noise ratio as a function of the input noise intensity. The origin of the additional maxima, which occur for small noise intensities, can be related to the structure of the interactions, thus the observed phenomenon is an example of structural stochastic multiresonance.

PACS: $05.45-\mathrm{a}, 75.30 \mathrm{Ds}, 76.50+\mathrm{g}$

\section{Introduction}

Complex networks of interactions and influence are ubiquitous in the modern society and economics [1-3]. A characteristic feature of many such networks, with examples ranging from the network of actor cooperation to the world wide web to the Internet, is their hierarchical organization [3]. Hence, investigation of signal detection and transmission in systems with the structure of hierarchical networks can be of practical importance. In the present paper this problem is addressed from the point of view of stochastic resonance (SR), a phenomenon which occurs in certain nonlinear systems driven by a periodic signal and noise and is characterized by the enhancement of a periodic component of an output signal for non-zero noise intensity [4] (for review see $[5,6]$ ). In systems exhibiting SR the power spectrum density of the output signal consists of a peak at the frequency of the input signal superimposed on a broadband noise background. The quantity characterizing SR is usually the output signalto-noise ratio (SNR), defined as the ratio of the height of the above-mentioned peak to the height of the noise background. A characteristic feature of systems with SR is that the SNR shows maximum as a function of the noise intensity. In a variant of SR called stochastic multiresonance (SMR) the SNR exhibits even more than one maximum [7-15]. In certain systems consisting of units located in the nodes and interacting along the edges of a complex network the origin of the multiple maxima of the SNR can be traced back to the structure of interactions, thus the corresponding phenomenon is called structural SMR [13-15]. In particular, structural SMR has been recently demonstrated in a system of threshold elements on a simple tree-like hierarchical network [15]. In this paper the latter results are first briefly reviewed in order to explain the role of the network structure in the occurrence of the multiple maxima of the SNR. Then it is shown that under similar assumptions structural SMR occurs also in systems of threshold elements on hierarchical networks with more complex topologies, reflecting those found in real social and economic networks.

The systems under study consist of $N$ non-dynamical threshold elements (artificial neurons) with the threshold $b$, i.e., elements with no internal dynamics, whose response to the external stimulus $z$ is given by the Heaviside unit step function $\Theta(z-b)$. The elements are driven by a common subthreshold periodic signal with amplitude $A<b$ and frequency $\omega_{s}$ and independent noise sources with intensity $D$. They are placed in the nodes of hierarchical networks and interact (in a way typical of artificial neural networks) along its edges. The edges can be directed, corresponding to one-way interactions, or undirected, corresponding to mutual interactions; the connection weight is denoted by $w$. The output $y^{(j)}(t)$ of the element in the node $j$ at a discrete time step $t$ is in general given by

$$
\begin{aligned}
& y^{(j)}(t)=\Theta\left[A \sin \omega_{s} t+D \eta^{(j)}(t)+\right. \\
& \left.\quad \frac{w}{k(j)} \sum_{m \in \mathcal{K}_{j}} y^{(m)}(t-1)-b\right], \quad j=1,2, \ldots N,
\end{aligned}
$$

where $\left\langle\eta^{(j)} \eta^{\left(j^{\prime}\right)}\right\rangle=\delta_{j, j^{\prime}}, k(j)$ is the degree of the node $j$, i.e., the number of incoming edges attached to the node $j$, and $\mathcal{K}_{j}$ denotes the neighbourhood of the node $j$, i.e., the set of all nodes linked to the node $j$ by edges. It is known that SR can be observed in a single threshold element driven by a subthreshold periodic signal and noise [16]. If the element is placed in a node of a regular network the maximum value of the SNR from its output signal can be increased due to interactions with other elements [17], which is an example of array-enhanced SR [18]. In contrast, the elements in the nodes of the hierarchical network can exhibit SMR (double maxima of the 
SNR) in a narrow range of parameters such as the amplitude of the periodic signal and weight of connections, depending on their position in the hierarchy and on the particular topology of connections.

\section{Structural stochastic multiresonance in the analytically solvable model}

\subsection{The model and methods of analysis}

Let us first recollect the results obtained in a simple model belonging to the class described by Eq. (1) in which the occurrence of the structural SMR can be confirmed both numerically and analytically [15]. The threshold elements are placed in the nodes of a hierarchical network with a tree-like structure which consists of $r+1$ layers, with $l$-th layer $(l=0,1,2, \ldots r)$ containing $n^{r-l}$ nodes, where $n$ is the branching factor (Fig. 1).

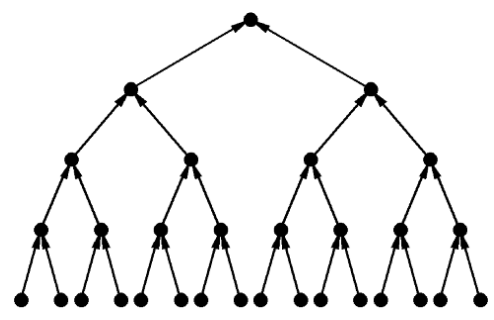

Fig. 1. A hierarchical network with a tree-like structure and directed edges, with $r=4$ and $n=2$. The layers $l=0,1,2, \ldots r$ are numbered from bottom $(l=0)$ to top $(l=r)$, and the nodes within the $l$-th layer from left $(i=1)$ to right $\left(i=n^{r-l}\right)$.

The edges of the network are directed, so that groups of $n$ nodes in the layer $l-1$ are connected to one node in the layer $l$, and correspond to non-zero unidirectional interactions between the threshold elements. The output $y_{l}^{(i)}$ of the element in the node $i$ belonging to the layer $l$ at a discrete time step $t$ is given by the appropriate variant of Eq. (1),

$$
\begin{gathered}
y_{0}^{(i)}(t)=\Theta\left[A \sin \omega_{s} t+D \eta_{0}^{(i)}(t)-b\right], \\
i=1,2, \ldots n^{r} ; \\
y_{l}^{(i)}(t)=\Theta\left[A \sin \omega_{s} t+D \eta_{l}^{(i)}(t)\right. \\
\left.+\frac{w}{n} \sum_{j=1}^{n} y_{l-1}^{(n(i-1)+j)}(t-1)-b\right] \\
l=1,2, \ldots r ; i=1,2, \ldots n^{r-l},
\end{gathered}
$$

where $\left\langle\eta_{k}^{(i)} \eta_{l}^{(j)}\right\rangle=\delta_{k, l} \delta_{i, j}$.

The SNR in the element $i$ belonging to the layer $l$ can be evaluated both numerically, from the time series from Eq. $(2,3)$, and analytically. The probability $P_{l}(t)$ that $y_{l}^{(i)}(t)=1$ for any element $i$ belonging to the lowest layer $l=0$ is

$$
P_{0}(t)=\operatorname{Pr}\left[A \sin \omega_{s} t+D \eta(t)-b>0\right]=
$$

$$
\frac{1}{2} \operatorname{erfc}\left[\frac{1}{D \sqrt{2}}\left(b-A \sin \omega_{s} t\right)\right],
$$

and to the layer $l=1,2, \ldots r$

$$
P_{l}(t)=
$$

$\sum_{k=0}^{n}\left(\begin{array}{l}n \\ k\end{array}\right) \Pi_{k}(t) P_{l-1}^{k}(t-1)\left[1-P_{l-1}(t-1)\right]^{n-k}$,

where $\Pi_{k}(t)$ is the conditional probability that $y_{l}^{(i)}(t)=1$ provided that $k$ of $n$ elements coupled to the element under study are excited,

$$
\begin{gathered}
\Pi_{k}(t)=\operatorname{Pr}\left[A \sin \omega_{s} t+D \eta(t)+w \frac{k}{n}-b>0\right] \\
=\frac{1}{2} \operatorname{erfc}\left[\frac{1}{D \sqrt{2}}\left(b-A \sin \omega_{s} t-w \frac{k}{n}\right)\right]
\end{gathered}
$$

(in Eqs. $(4,5) \eta(t)$ denotes white Gaussian noise with unit variance). The output SNR in the element belonging to the layer $l$ can be then evaluated as [16]

$$
\mathrm{SNR}=\frac{\left|\hat{P}_{l}\left(\omega_{s}\right)\right|^{2}}{\left\langle P_{l}(t)\right\rangle-\left\langle P_{l}^{2}(t)\right\rangle},
$$

where $\hat{P}_{l}(\omega)=T_{s}^{-1} \sum_{t=0}^{T_{s}-1} P_{l}(t) \exp (i \omega t)$, with $T_{s}=$ $2 \pi / \omega_{s}$, denotes the Fourier transform of $P_{l}(t)$, and the brackets denote the time average.

\subsection{Results and discussion}

Exemplary curves SNR vs. $D$ obtained from the output signals of the threshold elements in the consecutive layers of the network for fixed $r, n, w, b$ and small $\omega_{s}$ are shown in Fig. 2 for the case of a subthreshold periodic signal $A<b$. Theoretical curves were obtained from Eq. $(5)$ in the adiabatic approximation $P_{l}(t-1) \approx P_{l}(t)$ valid for $\omega_{s} \rightarrow 0$ and are in good agreement with numerical results. The SNR has at least one maximum as a function of the noise intensity, i.e., SR is observed. For $A \ll b$ usually there is only one maximum of the SNR located close to that for a single uncoupled element (Fig. 2(a)) and slightly higher, which is a signature of array-enhanced SR [18]. In contrast, for a narrow range of $A$ just below the threshold, depending on the connection weight $w$, additional maximum of the SNR appears at small $D$, which eventually becomes dominant for larger $l$, i.e., SMR occurs (Fig. 2(b)). From Fig. 3(a) it can be seen that for fixed $\omega_{s}, b, n$ double maxima of the SNR in the elements in the layers with $l>0$ occur if the amplitude of the periodic signal $A$ and the connection weight $w$ are within a triangle-like region whose size increases with $l$. Similarly, for fixed $l$ the size of this region increases with the branching factor $n$ (Fig. 3(b)). Although the triangle-like regions in Fig. 3 extend to the half-plane $A>b$, SMR is observed only for subthreshold periodic signals with $A<b$. For suprathreshold signals with $A>b$ SR disappears since the SNR diverges to infinity in the limit $D \rightarrow 0$ and the two maxima of the SNR, if present, are only local ones. 


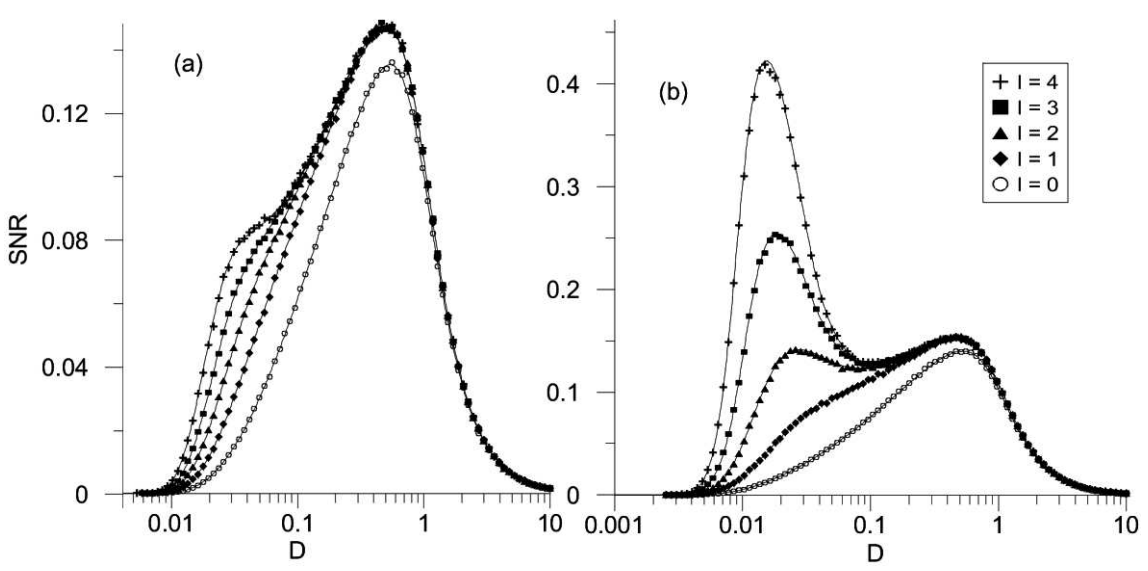

Fig. 2. SNR vs. the noise intensity $D$ from the output signal of the threshold elements in the consecutive layers $l=0,2, \ldots r$ of the tree-like network with directed edges, with $n=2, r=4$. Symbols - numerical results (see legend), solid curves - theoretical results from Eq. (7), $T_{s}=1024, b=1, w=0.07$ and (a) $A=0.976$, (b) $A=0.99$. The curve for $l=0$ matches that for a single uncoupled element.
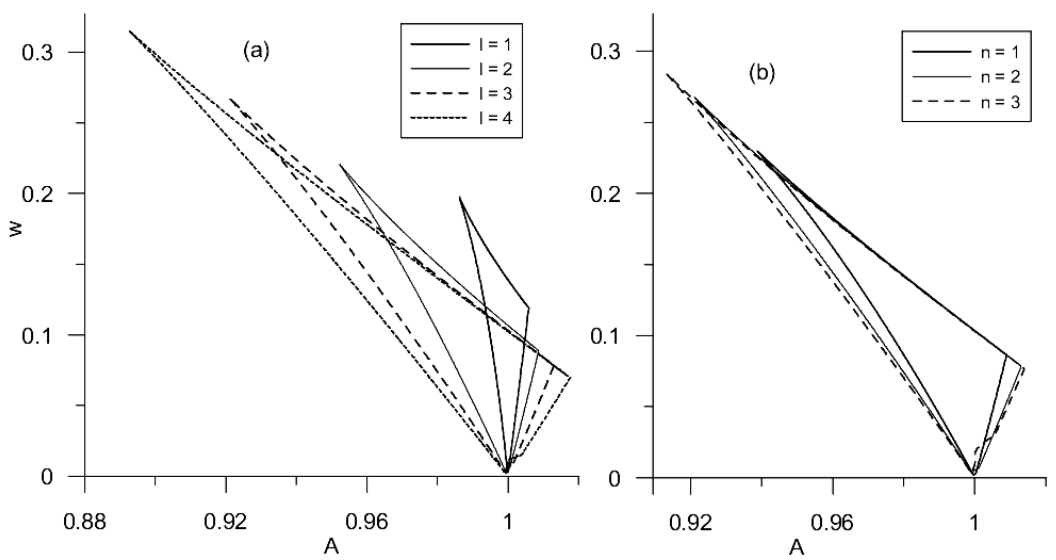

Fig. 3. Range of parameters $A, w$ where double maxima of the SNR from the output signal of the threshold elements in the consecutive layers $l=1,2, \ldots r$ of the tree-like network with directed edges are observed. The parameters are $T_{s}=1024, b=1$, (a) $r=4, n=2$ and various $l$ (see legend), (b) $r=3, l=3$ and various $n$ (see legends).

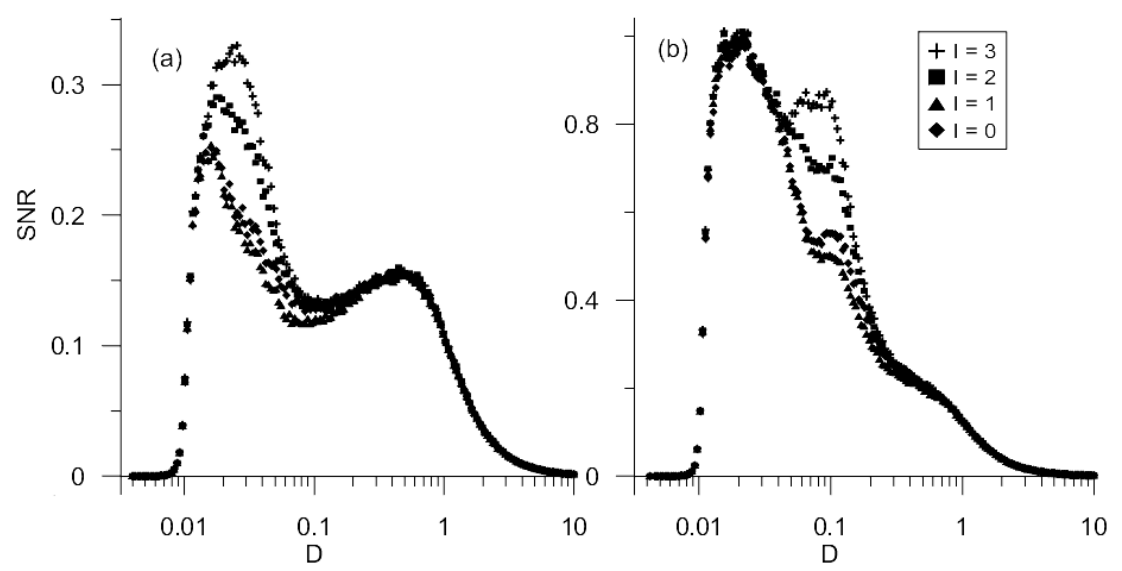

Fig. 4. Numerical results for the SNR vs. the noise intensity $D$ from the output signal of the threshold elements in the consecutive layers $l=0,1,2,3$ (see legend) of the tree-like network with undirected edges, $n=2, r=3, T_{s}=1024$, $b=1, A=0.98$ and (a) $w=0.1$, (b) $w=0.3$.

The appearance of the additional maximum of the SNR at small noise intensities is related to the structure of the network and thus the observed phenomenon is an exam- ple of structural SMR. The origin of the above-mentioned maximum can be explained as follows. It was verified that this maximum diminishes and eventually disappears 
as $\omega_{s}$ is increased, thus SMR occurs only in the adiabatic limit of slowly varying periodic signals. Moreover, for any $l$ the necessary (but not sufficient) condition for the occurrence of this maximum is $A+w / n>b$ (Fig. $3(\mathrm{a}))$. If the amplitude $A$ is very close to the threshold $b$ then even for small $D$ there is a non-negligible probability that at least one element in the network is excited when the periodic signal reaches its maximum value, in particular if $r$, and thus the number of elements in the network, is large enough. The impulse from this element is then transmitted to the element in the upper layer which also in the next time step becomes excited with high probability, since the sum of the slowly varying periodic signal, which is still close to maximum, and the input from the elements in the lower layer exceeds the threshold. Moreover, the noise is weak, thus the probability that it assumes a negative value $\eta$ which is large enough to prevent the element from being excited (c.a., $\eta<(b-A-w / n) / D)$ is negligible. Thus, provided that $r \ll T_{s}$, the output signal from any excited element easily reaches the top node. The elements in the upper layers, in particular that in the top layer, receive and average signals from $n$ branches of the network and thus are excited in phase with the input periodic signal with significantly higher probability than a single threshold element. This leads to the amplification of the periodic component in the output signal from these elements which is observed in the output power spectra and thus to the increase of the SNR. However, as $D$ is further increased the noise can assume larger negative values and prevent the excitation of any element, thus hampering the propagation of the impulses in any branch of the network. This results in the decrease of the SNR in the elements in the upper layers. Hence, the mechanism leading to the occurrence of the first maximum of the SNR is different from that typical of array enhanced SR, when all elements are synchronously excited in phase with the periodic signal [18]; the latter mechanism is responsible for the enhancement of the second maximum of the SNR.

\section{Structural stochastic multiresonance in other models}

3.1 The tree-like network with undirected edges

A direct extension of the model considered in Sec. 2 is the tree-like network with undirected edges corresponding to mutual interactions between threshold elements in the nodes. In this case a closed system of equations for $P_{l}(t)$, similar to Eqs. (4-6), cannot be written, thus the SNR from the output signal of the elements in the consecutive layers cannot be easily obtained analytically. Numerical simulations show that structural SMR can be observed in this system, too, under similar assumptions as in the case of the unidirectional interactions, i.e., for a slightly subthreshold, slowly varying periodic signal (Fig. 4). However, the dominant peak at small noise intensities occurs not only in the SNR from the output signals of the elements in the upper layers, with $l>0$, but also of the elements in the lowest layer, $l=0$. The reason is probably that the output signals of the elements in the upper layers, which contain a strong periodic component, can propagate back to the elements in the $l=0$ layer, which leads to the increase of the SNR in their output signals. Besides, in some cases the first peak of the SNR seems to split in two maxima (Fig. 4(b)).

\subsection{The Ravasz-Barabási network}

The networks characterizing the organization of modern society and economics are of course more complex than simple tree-like structures considered in Sec. 2 and 3.1. For example, empirical studies of several such networks in Ref. [3] show that apart from being hierarchical they are scale-free, i.e., the distribution of the degrees of nodes $P(k)$ obeys a power scaling law, $P(k) \propto k^{-\gamma}$, and highly clustered, i.e., there is a high probability that if two nodes are connected to the same node then they are also mutually connected. Besides, these networks exhibit a modular structure, i.e., they consist of groups of densely connected nodes which have few links outside the group. A generic example of a network with all the above-mentioned properties was proposed by Ravasz and Barabási [3]. The network is constructed as follows. In the first step there is a cluster of five nodes located on the vertices and in the centre of a square and connected by the edges along its sides and diagonals. In the second step the cluster is replicated four times, and the nodes on the vertices are connected to the central node of the old cluster. In the third step, the network generated in the second step is again replicated four times, and the nodes on the vertices of the peripherial squares of the four replicas are connected to the central node of the network generated in the second step. This leads to a complex network shown in Fig. 5, but the replication and connection steps can be repeated further. The Ravasz-Barabási network combines the hierarchical structure, modularity (the basic modules are densely connected clusters of five nodes on squares, created in the first step; the sets of five basic modules such as the network created in the second step are more sparsely connected modules; etc.), scale-free topology (with the exponent $\gamma=1+\ln 5 / \ln 4=2.161 \ldots$ ) and high clustering coefficient.

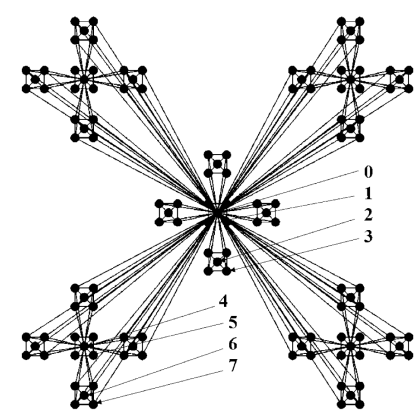

Fig. 5. The Ravasz-Barabási network after the third step of construction; the numbers denote nodes in which the SNR from the output signal of the threshold elements was evaluated from simulations (see Fig. 6).

In order to investigate SR in the system with the structure of the Ravasz-Barabási network (after three steps of 


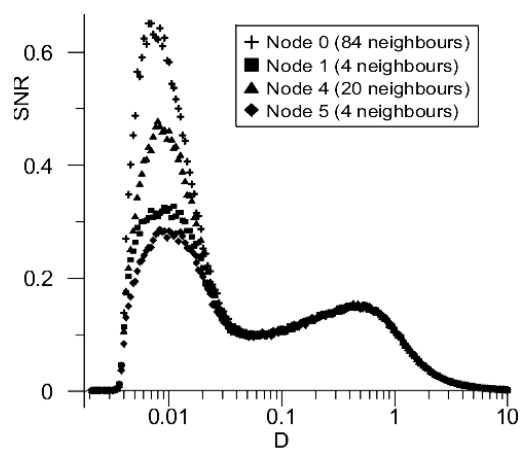

Fig. 6. Numerical results for the SNR vs. the noise intensity $D$ from the output signal of the threshold elements in selected nodes of the Ravasz-Barabási network (see legend; the location of the numbered nodes is shown in Fig. 5), $T_{s}=1024, b=1, A=0.99, w=0.05$.

construction) the threshold elements are placed in the nodes and connected along the edges by mutual interactions. Under similar assumptions as in the case of the tree-like networks (slowly varying periodic signal with the amplitude just below the threshold) SMR is observed in the numerical simulations. The SNR from the output signal of the threshold elements exhibits double maxima, and the peak at small noise intensity $D$ becomes dominant as the signal amplitude $A$ approaches the threshold $b$ from below, in particular in the case of nodes placed higher in the hierarchy (Fig. 6). It should be noted that the degree of the node is not the only factor determining the SNR from the output signal of the corresponding threshold element, but its position in the hierarchy is also important (cf. Fig. 6, where curves SNR vs. $D$ differ for the elements in the nodes 1 and 5 with the same degree). This suggests that the mechanism leading to the occurrence of the SMR resembles that in systems with the structure of the tree-like networks. If the periodic signal is slightly subthreshold and the noise is small but non-zero the random unit pulses generated by the elements low in the hierarchy propagate easily to the elements higher in the hierarchy. The latter elements receive and average signals from many modules of the network, thus the periodic component of their output signals is amplified.

\section{Summary and conclusions}

Structural SMR was observed in systems of nondynamical threshold elements placed in the nodes of hierarchical networks and interacting along their edges, which were driven by a common slowly varying periodic signal and independent noise sources. Results were presented for the tree-like network with unidirectional and mutual interactions as well as the Ravasz-Barabási network combining hierarchical and modular structure with properties typical of real networks such as scalefree topology and high clusterization. Double maxima of the SNR evaluated from the output signal of certain elements, in particular those located high in the hierarchy, occur for a narrow range of parameters such as the strength of connections and the amplitude of the periodic signal, which must be close to the threshold. The mechanism leading to the appearance of the additional maximum at small noise intensities is based on averaging of inputs received by the elements located high in the hierarchy from many branches or modules of the network.

The obtained results suggest that structural SMR may be a widespread phenomenon in complex systems with hierarchical structure. In particular, the existence of a dominant maximum of the SNR at small noise intensities may facilitate the detection of subthreshold informationcarrying signals in weakly fluctuating environment by units located high in the hierarchy (e.g., top managers in the hierarchical company structure).

\section{Acknowledgements}

This paper was supported by the Polish Ministry of Science, Grant No. 496/N-COST/2009/0

\section{References}

[1] R. Albert and A.-L. Barabási, Rev. Mod. Phys. 74, 47 (2002).

[2] S. N. Dorogovtsev, J. F. F. Mendes, Evolution of Networks. From Biological Nets to the Internet and $W W W$, Oxford University Press, Oxford, UK, 2003.

[3] E. Ravasz and A. Barabási, Phys. Rev. E67, 026112 (2003).

[4] R. Benzi, A. Sutera, and A. Vulpiani, J. Phys. A14, L453 (1981).

[5] L. Gammaitoni, P. Hänggi, P. Jung, and F. Marchesoni, Rev. Mod. Phys. 70, 223 (1998).

[6] M. D. McDonell, N. G. Stocks, C. E. M. Pearce, and D. Abbott, Stochastic Resonance. From Suprathreshold Stochastic Resonance to Stochastic Signal Quantization, 1st ed. (Cambridge University Press, Cambridge, 2008).

[7] J. M. G. Vilar and J. M. Rubí, Phys. Rev. Lett. 78, 2882 (1997).

[8] J. M. G. Vilar and J. M. Rubí, Physica A264, 1 (1999).

[9] S. Matyjaśkiewicz, A. Krawiecki, J. A. Ho_lyst, and L. Schimansky-Geier, Phys. Rev. E68, 016216 (2003).

[10] M. Evstigneev, P. Reimann, V. Pankov, and R. H. Prince, Europhys. Lett. 65, 7 (2004).

[12] E. I. Volkov, E. Ullner, and J. Kurths, Chaos 15 , 023105 (2005).

[12] R. Mankin, K. Laas, T. Laas, E. Reiter, Phys. Rev. E78, 031120 (2008).

[13] A. Krawiecki, Acta Phys. Polonica B39, 1103 (2008).

[14] A. Krawiecki, Eur. Phys. J. B69, 81 (2009).

[15] M. Kaim and A. Krawiecki, Phys. Lett. A374, 4814 (2010).

[16] F. Chapeau-Blondeau, Phys. Rev. E53, 5469 (1996).

[17] A. Krawiecki, A. Sukiennicki, and R.A. Kosiński, Phys. Rev. E62, 7683 (2000).

[18] J. F. Lindner, B. K. Meadows, W. L. Ditto, M. E. Inchiosa, and A. R. Bulsara, Phys. Rev. Lett. 75, 3 (1995). 